\title{
Gestational Length Affects a Change in the Transepithelial Voltage and the rNKCC2 Expression Pattern in the Ascending Thin Limb of Henle's Loop
}

\author{
MINAKO NISHINO, TETSUJI MORIMOTO, TOSHIYUKI NISHIO, ULVIYYA F. ASLANOVA, ELNUR I. FARAJOV, \\ NAONORI KUMAGAI, NORIKO SUGAWARA, SHORI TAKAHASHI, ATSUSHI OHSAGA, YOSHIO MARUYAMA, \\ SHIGERU TSUCHIYA, AND YOSHIAKI KONDO
}

\begin{abstract}
Department of Pediatrics [M.N., T.M., T.N., U.F.A., N.K., N.S., S. Tsuchiya], Department of Physiology [A.O., Y.M.], Department of Medical Informatics [E.I.F., Y.K.], Tohoku University Graduate School of Medicine, Sendai, Japan 980-8574; Department of Pediatrics [S. Takahashi.], Nihon University School of Medicine, Tokyo, Japan 101-8309
\end{abstract}

\begin{abstract}
To examine whether the functional and morphologic conversion of the neonatal ascending thin limb (ATL) of Henle's loop is related to gestational length, we evaluated the transepithelial voltages (Vts) of ATLs in perinatal mouse, hamster, rabbit, and rat kidneys. In isolated microperfused tubule preparations, Vts of neonatal ATLs were $23.8 \pm 1.4$ in mouse, $25.7 \pm 2.2$ in hamster, and $18.2 \pm 1.6 \mathrm{mV}$ in rabbit. The influence of gestational length on the $\mathrm{Vts}$ and rat $\mathrm{Na}-\mathrm{K}-\mathrm{Cl}$ cotransporter (rNKCC2) expression pattern was also examined in perinatal rats subjected to a prolonged gestation due to either a daily s.c. injection of $5 \mathrm{mg}$ progesterone or ligation of the extremities of the uterine horn. Vts of $\mathrm{d} 3$ neonates were $2.9 \pm 1.0$ $(p<0.0001$ versus $\mathrm{d} 0$ ); Vts of $\mathrm{d} 23$ fetuses subjected to ligation were $4.9 \pm 0.8(p<0.005$ versus $\mathrm{d} 0)$; and Vts of $\mathrm{d} 23$ fetuses given progesterone were $3.4 \pm 1.7 \mathrm{mV}$ ( $p<0.001$ versus $\mathrm{d} 0$ ). $\mathrm{rNKCC} 2$ expression tended to disappear in the renal papillae of d 23 fetuses. Our data demonstrate that the perinatal conversion of the ATL is a phenomenon commonly observed among rodents; furthermore, it is dependent on the gestational length, but unrelated to the birth process. (Pediatr Res 61: 171-175, 2007)
\end{abstract}

$\mathrm{M}^{\mathrm{a}}$ ammalian neonates possess a nearly mature urinediluting ability at birth (1); however, human neonates are unable to produce hypertonic urine at concentrations $>400$ $\mathrm{mOsmol} / \mathrm{kg}$, and rats are unable to produce hypertonic urine $>600 \mathrm{mOsmol} / \mathrm{kg}$. These observations indicate that the urineconcentrating ability is quite immature at birth (2). A number of researchers have tried to determine why the urineconcentrating ability of neonates does not reach adult levels; however, none have succeeded in explaining this phenomenon.

It was first reported in the 19th century that morphologically, neonatal kidneys do not possess inner medullae (3). Subsequently, Kim et al. (4) clarified the subject by reporting that the ATL of Henle's loop of neonatal rats is also morphologically different from that of adults. They reported that the

Received April 5, 2006; accepted September 20, 2006.

Correspondence: Tetsuji Morimoto, M.D., Ph.D., Department of Pediatrics, Tohoku University Graduate School of Medicine, 1-1 Seiryo-machi, Aoba-ku, Sendai, Japan 980-8574; e-mail: morimoto-thk@umin.ac.jp

This work was supported in part by a grant-in-aid from the Ministry of Education, Science, and Culture in Japan and by a research grant 02C6 from the Salt Science Research Foundation in Japan.

DOI: $10.1203 / p d r .0 b 013 e 31802 d 7791$
ATL in the neonatal period is very similar to the thick ascending limb (TAL) and that the tubule is replaced by the mature ATL cells after apoptosis and transformation.

In an attempt to clarify the urine-concentrating mechanism in the neonatal period, we examined the renal tubular function directly in a series of experiments using in vitro tubule microperfusion technique and measured mRNAs for wellknown transporters and channels in the isolated inner medullary tubules of fetal, neonatal, and adult rat kidney (5). Our results led to a striking new concept regarding the maturating mechanism for the urine concentrating ability of mammalian neonates. We demonstrated that the renal medullary tubule organization of neonatal rats shares a marked similarity to the avian renal medulla. We postulate that the neonatal period is a phase of functional conversion of the urine concentrating mechanism from avian type to the mammalian type.

In mammals, a urine-concentrating mechanism that is more dependent on urea than $\mathrm{NaCl}$ is the major protective response to a dry environment. In birds, both regulation of glomerular filtration and a urine-concentrating mechanism that is dependent on $\mathrm{NaCl}$ play important roles in fluid and electrolyte homeostasis. From the phylogenetic perspective, the urineconcentrating mechanism is unique in that it is only present in the metanephros of birds and mammals. Urine-concentrating ability develops after birth and matures during the weaning period.

Changes in the renal medullary tubular transport properties of mature quail (6-9), neonatal rats, and mature rats were compared in our previous report (5). As we have previously indicated, the functional organization of the medullary tubules in neonatal rats is very similar to that of the mature quail in that the entire Henle's loop is impermeable to water, and the entire ascending limb actively reabsorbs $\mathrm{NaCl}$. It is also remarkable that the neonatal ATL and inner medullary collecting duct is poorly permeable to urea, indicating minor contribution of urea to the neonatal urine-concentrating mechanism in rats. The conversion of the inner medullary tubular function in the neonatal rat is therefore regarded as the

Abbreviations: ATL, ascending thin limb; rNKCC2, rat Na-K-Cl cotransporter; TAL, thick ascending limb; Vt, transepithelial voltage 
a. Repeated subcutaneous injection of progesterone to pregnant rats

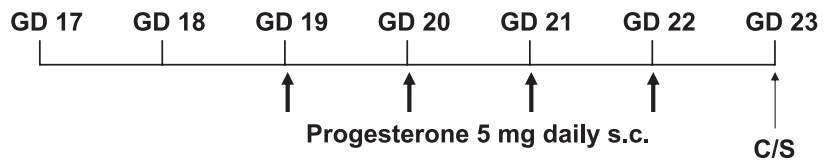

b. Uterine horn ligation in pregnant rats

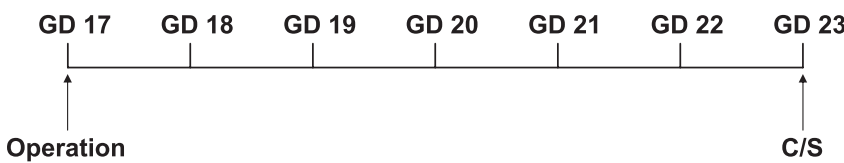

Figure 1. Experimental protocol. Five milligrams of progesterone was s.c. injected into maternal rats daily from gestational $d 19$. The extremities of the uterine horns were ligated on gestational d 17. The fetuses were harvested by cesarean section on gestational d 23 .

replacement of transport properties from simple $\mathrm{NaCl}$ accumulation to complex $\mathrm{NaCl}$ and urea accumulation for the urine concentrating mechanism.

The aim of the present study was to determine that the Vt in the ATL is positive in other rodents besides the neonatal rat and to test whether prolonged gestation affects a maturational change of the NKCC2 expression pattern in the rat kidney.

\section{METHODS}

Animals. Pregnant New Zealand white rabbits, Sprague-Dawley rats, C57BL/6 mice, and golden hamsters were obtained from Japan SLC, Inc., Hamamatsu, Japan. All animals were allowed free access to water and chow. This study was carried out in accordance with the Guide for Animal Experiment, Tohoku University and Tohoku University School of Medicine.

Prolonged gestation. We used two methods to induce a prolonged gestation (Fig. 1): (1) Daily s.c. injections of $5 \mathrm{mg}$ progesterone in sesame oil to the maternal rats from d 19 through 23 of gestation (progesterone method) (10); (2) on the 17th day of gestation, the animals were subjected to an abdominal incision and ligature of the extremities of the uterine horn under ether anesthesia (ligation method) (11). The fetuses were harvested by cesarean section on gestational d 23 .

Isolation and microperfusion of renal tubules. Each rat (d 23 fetus and d 3 neonate) was anesthetized with intraperitoneal pentobarbital, and the left kidney was removed. The renal tubule segment was microdissected with fine forceps under a stereoscopic microscope. The border between the outer and inner medulla in neonatal kidneys was identified macroscopically as previously reported by Kim et al. To avoid contamination of the tubules originating from the outer medulla, the inner two thirds of the inner medulla in neonatal kidneys was used for microdissection of the tubule fragments. Furthermore, the tubules were dissected from the cortical side and special care was taken to obtain the tubules containing the Henle's loop. To maximize the reproducibility of this isolation, relatively short segments $(0.5-0.8 \mathrm{~mm})$ were excised. A fragment of the tubule was transferred to a chamber on the stage of an inverted microscope and was microperfused in vitro at $37^{\circ} \mathrm{C}$ using the Burg et al. method (12) with some modification. Both sides of the tubule were microperfused with $N$-2-hydroxyethylpiperazine- $N^{1}$-2-ethanesulfonic acid (HEPES)-buffered Ringer's solution containing (in mmol/L) $135 \mathrm{NaCl}, 3.0$ $\mathrm{KCl}, 2.0 \mathrm{KH}_{2} \mathrm{PO}_{4}, 1.5 \mathrm{CaCl}_{2}, 1.0 \mathrm{MgCl}_{2}, 10 \mathrm{HEPES}, 100$ urea, 1.0 sodium acetate, 5.5 glucose, and 5.0 L-alanine. The solution was titrated to $\mathrm{pH} 7.4$ with $\mathrm{NaOH}$ at $37^{\circ} \mathrm{C}$. The perfusion rate of the luminal solution in the studies for spontaneous $\mathrm{Vt}$ measurements was kept at $<2 \mathrm{~nL} / \mathrm{min}$. The specimen was discarded if tubular damage was observed microscopically.

Measurement of transepithelial potentials. The Vt was measured by connecting the bath and perfusion pipette via a saturated $\mathrm{KCl}$ flowing boundary and a $\mathrm{NaCl}$ Ringer's-containing agar bridge. A high-input impedance electrometer was used to monitor the Vts (Duo 773; WP Instruments, New Haven, CT). We used a rapid bath flow system that allowed us to exchange the entire bath volume within $1 \mathrm{~s}$. The $\mathrm{KCl}$ flowing boundary minimized the changes in the liquid junction potential due to the solution exchange (13).
Immunohistological analyses. The kidneys from each group $(\mathrm{d} 0, \mathrm{~d} 3$, and ligation) were removed quickly and then immersed with $4 \%$ paraformaldehyde in phosphate-buffered saline (PBS) overnight at $4{ }^{\circ} \mathrm{C}$. Fiftymicrometer sections were cut transversely through the entire kidney with a MICROSLICER DTK-1000 (E.M. Douhan, Kyoto, Japan). Immunostaining was performed using a TSA-direct kit (Perkin-Elmer Life Sciences, Boston, MA) according to the manufacturer's instructions. Briefly, 50- $\mu \mathrm{m}$ thick sections were washed with PBS three times for $5 \mathrm{~min}$. Before incubation with the primary antibody, all tissue sections were incubated for $30 \mathrm{~min}$ with TNB blocking buffer. After washing with PBS, the tissue sections were then incubated overnight at $4^{\circ} \mathrm{C}$ in rabbit antisera against rat NKCC2 (1:500) in TNB blocking buffer. After washing with TNT wash buffer, biotinylated secondary antibody in PBS was added for $30 \mathrm{~min}$ at room temperature. Subsequent steps were conducted according to the manufacturer's instructions. Ultimately, the sections were mounted on silane-coated glass slides (Dako, Kyoto, Japan). The sections were examined and photographed with a FLUOVIEW laser-scanning microscope (Olympus, Tokyo, Japan). The polyclonal antibody for rNKCC2 was kindly donated by Dr. Steven C. Hebert (14).

Chemicals. HEPES was obtained from Sigma Chemical Co. (St. Louis, MO). All other chemicals were purchased from Wako Pure Chemical Industries (Osaka, Japan).

Statistics. The data for the Vts represent the values obtained when the voltage stabilized for at least $30 \mathrm{~s}$. On rare occasions when the voltage did not stabilize, the maximal deflection was chosen as the representative value. The data shown are either original tracings or mean $\pm \mathrm{SE} ; n$ equals the number of tubule samples used for in vitro microperfusion. Comparisons between the two groups used the unpaired $t$ test. Significance was asserted with $p<0.05$.

\section{RESULTS}

Vts. The Vts were observed in the ATLs when the tubules were microperfused in vitro in the control HEPES-buffered solution. The results in three different animals are presented in Figure 2. The Vts were $23.8 \pm 1.4 \mathrm{mV}(n=5)$ on $\mathrm{d} 0$ mice, $25.7 \pm 2.2 \mathrm{mV}(n=4)$ on $\mathrm{d} 0$ hamsters, and $18.2 \pm 1.6 \mathrm{mV}$ $(n=3)$ on $\mathrm{d} 0$ rabbits. The $\mathrm{Vt}$ of the $\mathrm{d} 0$ rat was $13.8 \pm 1.4$ $\mathrm{mV}(n=18)$ in a previous study (5).

A representative tracing showing the $\mathrm{Vt}$ on the $\mathrm{d} 0$ rabbit is presented in Figure 3. The Vt had a flow dependency and was definitely positive. The results for each group are presented in Figure 4. The Vts were $13.8 \pm 1.4 \mathrm{mV}(n=18)$ on $\mathrm{d} 0$ neonates, $2.9 \pm 1.0 \mathrm{mV}(n=9)$ on d 3 neonates, $4.9 \pm 2.8$ $\mathrm{mV}(n=9)$ on $\mathrm{d} 23$ fetuses (ligation), and $3.4 \pm 1.7 \mathrm{mV}(n=$ 6) on d 23 fetuses (progesterone). We previously verified that spontaneously evoked Vts of the ATL on d 0 were positive

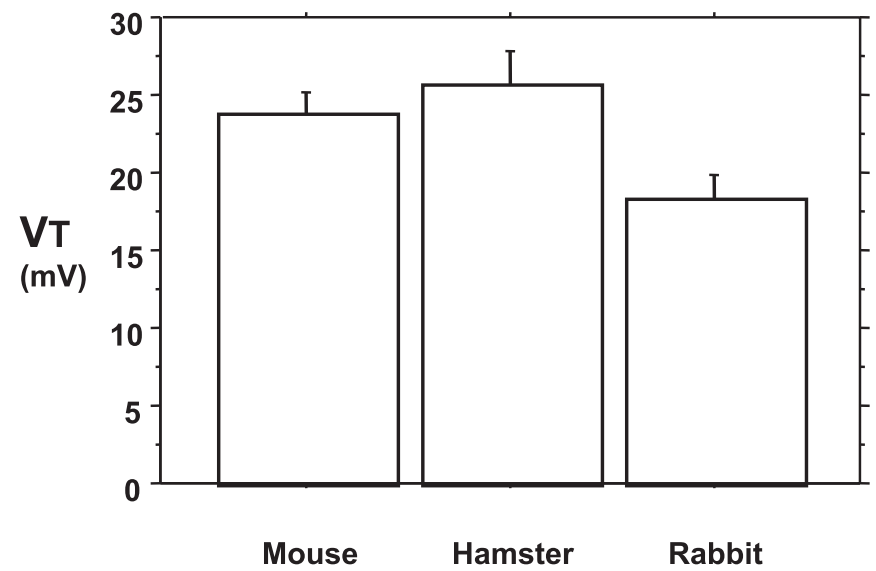

Figure 2. Vts of mice, hamsters, and rabbits on d 0 . Spontaneously evoked Vts of the ATL were observed in mice, hamsters, and rabbits on $\mathrm{d} 0$. The Vts were $23.8 \pm 1.4 \mathrm{mV}(n=5)$ in mice, $25.7 \pm 2.2 \mathrm{mV}(n=4)$ in hamsters, and $18.2 \pm 1.6 \mathrm{mV}(n=3)$ in rabbits. Spontaneously evoked Vts of mice, hamsters, and rabbits were all positive. 


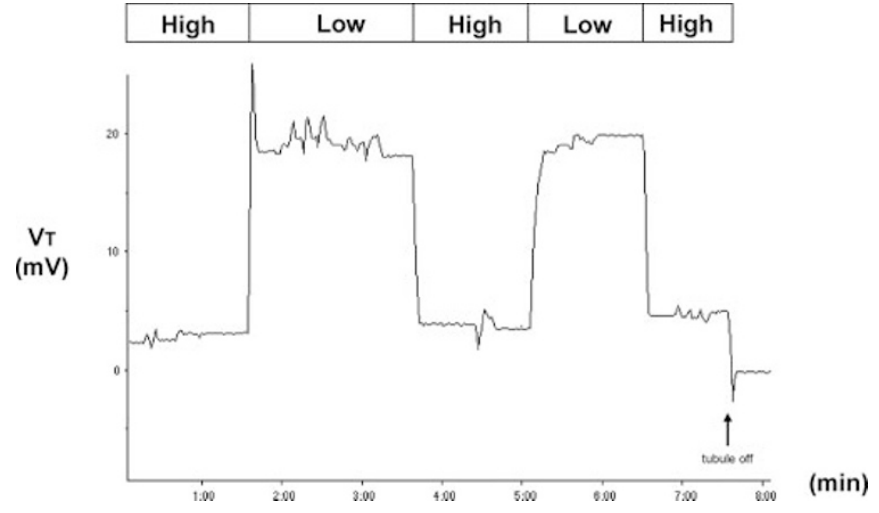

Figure 3. Representative tracing of the $\mathrm{d} 0$ rabbit. A flow-dependent transmural voltage was present. When the perfusion rate was reduced by lowering the height of the perfusate reservoir, the positive Vt increased. The Vt was approximately $+19 \mathrm{mV}$ in a steady-state condition at a low flow rate.

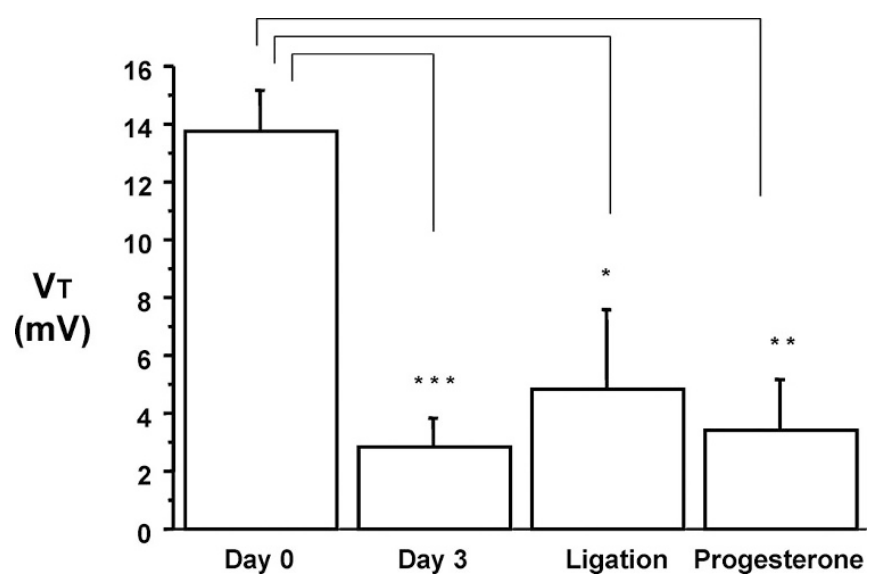

Figure 4. Transepithelial voltages in each group. Spontaneously evoked Vts of the ATL were observed in d 0 neonates, $\mathrm{d} 3$ neonates, $\mathrm{d} 23$ fetuses subjected to ligation, and d 23 fetuses given progesterone. The Vts were $13.8 \pm 1.4 \mathrm{mV}$ $(n=18)$ on $\mathrm{d} 0,2.9 \pm 1.0 \mathrm{mV}(n=9)$ on $\mathrm{d} 3,3.4 \pm 1.7 \mathrm{mV}(n=6)$ on $\mathrm{d}$ 23 (progesterone), and $4.9 \pm 2.8 \mathrm{mV}(n=9)$ on d 23 (ligation). ${ }^{*} p<0.005$; $* * p<0.001 ; * * * p<0.0001$ (vs d 0 ).

and that the neonatal ATL possessed an active $\mathrm{Na}^{+}$reabsorption mechanism similar to that of the mature TAL. Furthermore, the spontaneous positive Vts diminished daily and disappeared by $\mathrm{d} 5$.

The Vts of $\mathrm{d} 3$, ligation, and progesterone groups were markedly decreased, when compared with that of the d 0 rat. The changes in Vts were statistically significant $(p<0.0001, \mathrm{~d} 0$ versus $\mathrm{d} 3 ; p<0.001, \mathrm{~d} 0$ versus progesterone; and $p<0.005$, $\mathrm{d} 0$ versus ligation). However, there are no significant differences between the $\mathrm{d} 3$, ligation, and progesterone groups.

To characterize the active electrical properties of the ATL on d 23 fetuses prolonged by ligation and progesterone, the effects of bumetanide was examined. Bumetanide at 0.1 $\mathrm{mmol} / \mathrm{L}$ applied to the lumen significantly inhibited the Vts of the ATLs in the both groups. The Vts decreased from $1.2 \pm$ $0.4 \mathrm{mV}$ to $0.1 \pm 0.2 \mathrm{mV}$ in the progesterone group $(n=5$, $p<0.005$, paired $t$ test) and from $1.7 \pm 0.6 \mathrm{mV}$ to $-0.1 \pm$ $0.2 \mathrm{mV}$ in the ligation group ( $n=6, p<0.05$, paired $t$ test), respectively. These results indicate the presence of an active

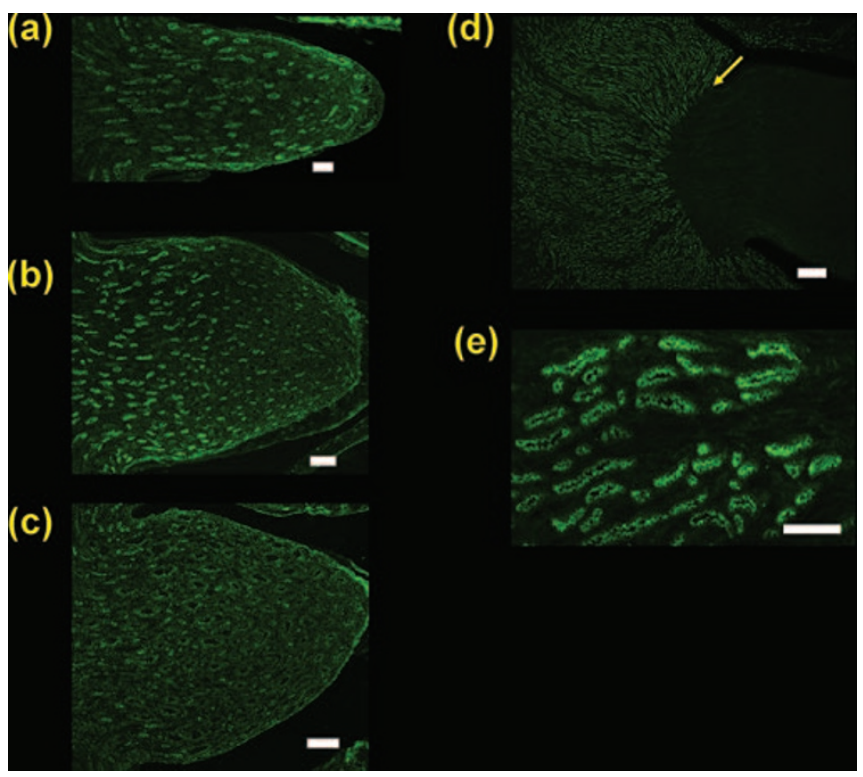

Figure 5. Immunohistochemistry. (a-e) The immunofluorescent staining for the rNKCC2 is depicted. Strong immunoreactivities of the rNKCC2 were present in the entire ascending limb of $\mathrm{d} 0$ renal papillae $(a)$. The $\mathrm{rNKCC} 2$ immunoreactivity disappeared from one third of the renal papilla on $\mathrm{d} 3(b)$. There was a little immunoreactivity in the renal papillae of $\mathrm{d} 23$ fetuses subjected to ligation $(c)$. The immunoreactivity for the $\mathrm{rNKCC} 2$ in the adult kidney was present mainly in the outer medulla $(d)$. The staining is limited to the luminal membranes $(e)$. The arrow indicates the border between outer and inner medulla. [Magnification: $\times 40$, bar: $100 \mu \mathrm{m}(a-d)$; $\times 100$, bar: $500 \mu \mathrm{m}(e)]$.

$\mathrm{NaCl}$ reabsorption mechanism in the ATL on d 23 fetuses prolonged by ligation and progesterone.

Immunofluorescent identification of $\mathrm{rNKCC} 2$ in the ascending limb. Rat NKCC2 was identified in immunofluorescent studies using polyclonal antibody for rNKCC2 (Fig. 5). The strong immunoreactivity of $\mathrm{rNKCC} 2$ was detected in the entire ascending limb of the $\mathrm{d} 0$ rat kidney and the cortical TAL of the adult rat kidney. At high magnification, the rNKCC2 protein was expressed in the apical membrane as previously reported; however, it disappeared from one third of the renal papilla on $\mathrm{d} 3$ neonates and almost the entire papilla on d 23 fetuses subjected to ligation. No immunostaining was present in the kidney control sections when preimmune rabbit serum was present for rNKCC2 (data not shown).

To quantify the changes in the number of rNKCC2-positive ATLs in the perinatal renal papilla, we counted the numbers of the fluorescein isothiocyanate fluorescent tubules in the distal two thirds of the longitudinal middle $50 \mu \mathrm{m}$ thick papilla slices from d 3 neonates from normal delivery and from d 23 fetuses with their gestation prolonged by ligation. The fluorescence-positive tubules on $\mathrm{d} 3$ neonates were much more frequent than those of the $\mathrm{d} 23$ fetus (104 \pm 3 versus $28 \pm 3$, $n=4, p<0.0001)$. These results clearly indicate that birth process is unrelated to the change of $\mathrm{rNKCC} 2$ expression pattern in developing kidney.

\section{DISCUSSION}

In this study, we have demonstrated that, in addition to the rat, the ATL of the mouse, rabbit, and hamster kidneys have 
a positive voltage on d 0 . Conversely, Imai et al. previously reported that the Vts of the ATL in the adult hamster and mouse were essentially zero (15). We previously reported that the ATL of the neonatal rat possessed a positive voltage on d 0 , which decreased to nearly zero by d 14 . We also demonstrated that this voltage is blocked by bumetanide in the luminal side and ouabain in the basolateral side. Bumetanide is an inhibitor of $\mathrm{Na}-\mathrm{K}-2 \mathrm{Cl}$ cotransporter in the TAL (16), and ouabain is a specific inhibitor of the ubiquitous $\mathrm{Na}^{+}$pump in mammalian cell membranes (17). A dramatic difference was found between the ATL and TAL; the Vts are present during neonatal period in both segments and disappear during the weaning period only in the ATL. Because the bumetanide-sensitive Vts in the TAL is the result of active $\mathrm{NaCl}$ reabsorption, these observations indicate that the neonatal rat ATL possesses an active $\mathrm{NaCl}$ reabsorption mechanism as does the TAL.

In this study, we observed that the Vts decreased when the flow rate was changed from slow to fast. Satlin et al. $(18,19)$ previously reported that flow-dependent $\mathrm{Na}$ absorption and $\mathrm{K}$ secretion are observed in the microperfused rabbit CCDs. To the best of our knowledge, ENaC and Maxi-K channels do not express in the rat ATL and TAL segments. Furthermore, it is not known whether some other flow-dependent channels are present. Further studies are indicated to elucidate this phenomenon.

In addition, immunohistochemistry has revealed that rNKCC2 protein is expressed in the entire renal papilla of the d 0 rat. However, the expression pattern changed daily to that of the adult rat and eventually disappeared from the inner medullary area. Kaplan et al. (14) previously verified that immunofluorescence studies using this polyclonal antibody on adult rat kidney sections showed an intense signal limited to the apical surfaces of the medullary TAL and the cortical TAL segments. We also demonstrated the same expression pattern of $\mathrm{rNKCC} 2$ in a previous study. Our results indicate that developmental changes are present in the urine-concentrating mechanism of rodents (mouse, hamster, and rabbit) during postnatal maturation. Taken together, we postulate that there is a qualitatively distinct phase in the organization of the inner medulla early in the neonatal period of these rodents.

What is the trigger for this maturational change? Kim et al. (4) reported that the cuboidal epithelium of the TAL of the renal papilla is gradually transformed into the ATL during the first 2 weeks of life. However, despite this study, little is currently known about a trigger for this structural change. We examined whether the gestational length affected this maturational change. Artificially prolonged gestation induced by daily s.c. progesterone injections has been undertaken by numerous investigators (10). We also used a surgical technique to prolong gestation to rule out the possible effect of exogenous progesterone. This study demonstrated that the $\mathrm{Vt}$ of each group was still positive; however, it was drastically decreased when compared with that of the $\mathrm{d} 0$ rat. There were no significant differences with respect to the Vts between the three groups (d 3, fetus d 23 progesterone, and fetus d 23 ligation). Moreover, the expression of rNKCC2 in d 23 fetuses subjected to ligation decreased when compared with that of d 3 neonates. An intrauterine high pressure might affect the rNKCC2 expression pattern.

These observations strongly suggest that prolonged gestation alter the Vts and the expression pattern of rNKCC2.

The factors that are responsible for the postnatal maturation of urine-concentrating mechanism are currently unclear. Apoptosis plays an important role in rat kidney morphogenesis. Kim et al. (4) reported that apoptosis in the renal papilla participates in the elimination of the thick ascending epithelial cells and in the formation of the ATL epithelium. However, no reports exist in the literature that elucidate a factor regulating the maturational change of the urine-concentrating mechanism. Baum et al. (20) reported that glucocorticoids affect maturation of $\mathrm{Na}^{+} / \mathrm{H}^{+}$exchanger 3 (NHE3) during renal cortical development. According to their study, daily s.c. administration of dexamethasone resulted in a twofold increase in NHE3 mRNA abundance and threefold increase in NHE3 protein abundance. This is of interest because it is well-known that the perinatal increase in glucocorticoid concentration is an important factor in the maturation of several organs, including the lung, liver, and intestine (21-23). Nevertheless, further research is required to elucidate a trigger for this maturational change.

In conclusion, we demonstrated that the ATL of rodents (mouse, hamster, and rabbit) also exhibits a positive transepithelial voltage on $\mathrm{d} 0$. Furthermore, this study demonstrated that $\mathrm{rNKCC} 2$ expression of $\mathrm{d} 3$ neonates and d 23 fetuses subjected to ligation decreased in the renal papillae. The factors responsible for the postnatal change in the urineconcentrating mechanism are currently unknown.

Acknowledgments. The authors thank Naoko Sato for her technical assistance.

\section{REFERENCES}

1. Spitzer A, Schwartz G 1992 The kidney during development. In: Windhager E (ed) Handbook of Physiology: Renal Physiology. Oxford University Press, New York, pp 475-544

2. Edwards BR, Mendel DB, LaRochelle FT Jr, Stern P, Valtin H 1982 Postnatal development of urinary concentrating ability in rats: changes in renal anatomy and neurohypophysial hormones. In: Spitzer A (ed) The Kidney During Development: Morphology and Function. Masson, New York, pp 233-240.

3. Hamburger O 1890 Uber dir Entwicklung der Saugetierniere. Arch Anat Physiol suppl: $15-51$

4. Kim J, Lee GS, Tisher CC, Madsen KM 1996 Role of apoptosis in development of the ascending thin limb of the loop of Henle in rat kidney. Am J Physiol 271:F831F845

5. Liu W, Morimoto T, Kondo Y, Iinuma K, Uchida S, Imai M 2001 Avian-type" renal medullary tubule organization causes immaturity of urine-concentrating ability in neonates. Kidney Int 60:680-693

6. Nishimura H, Imai M, Ogawa M 1986 Diluting segment in avian kidney. I. Characterization of transepithelial voltages. Am J Physiol 250:R333-R340

7. Nishimura H, Koseki C, Imai M, Braun EJ 1989 Sodium chloride and water transport in the thin descending limb of Henle of the quail. Am J Physiol 257:F994F1002

8. Nishimura H, Koseki C, Patel TB 1996 Water transport in collecting ducts of Japanese quail. Am J Physiol 271:R1535-R1543

9. Nishimura H, Fan Z 2002 Sodium and water transport and urine concentration in avian kidney. Symp Soc Exp Biol 129-151

10. Thliveris JA 1976 Fine structure of the placental labyrinth in the rat at term and during prolonged gestation. Virchows Arch B Cell Pathol 21:169-178

11. Faridy EE 1981 Fetal lung development in surgically induced prolonged gestation. Respir Physiol 45:153-166

12. Burg M, Grantham J, Abramow M, Orloff J 1966 Preparation and study of fragments of single rabbit nephrons. Am J Physiol 210:1293-1298 
13. Kondo Y, Fromter E 1987 Axial heterogeneity of sodium-bicarbonate cotransport in proximal straight tubule of rabbit kidney. Pflugers Arch 410:481-486

14. Kaplan MR, Plotkin MD, Lee WS, Xu ZC, Lytton J, Hebert SC 1996 Apical localization of the Na-K-Cl cotransporter, rBSC1, on rat thick ascending limbs. Kidney Int 49:40-47

15. Imai M, Kusano E 1982 Effects of arginine vasopressin on the thin ascending limb of Henle's loop of hamsters. Am J Physiol 243:F167-F172

16. Haas M, Forbush B 3rd 1998 The Na-K-Cl cotransporters. J Bioenerg Biomembr 30:161-172

17. Farman N $1996 \mathrm{Na}, \mathrm{K}-$ pump expression and distribution in the nephron. Miner Electrolyte Metab 22:272-278

18. Satlin LM 1994 Postnatal maturation of potassium transport in rabbit cortical collecting duct. Am J Physiol 266:F57-F65
19. Satlin LM, Sheng S, Woda CB, Kleyman TR 2001 Epithelial Na+ channels are regulated by flow. Am J Physiol Renal Physiol 280:F1010-F1018

20. Baum M, Biemesderfer D, Gentry D, Aronson PS 1995 Ontogeny of rabbit rena cortical NHE3 and NHE1: effect of glucocorticoids. Am J Physiol 268:F815-

21. Ballard PL 1979 Glucocorticoids and differentiation. In: Baxter JD, Rousseau GG (eds) Monographs on Endocrinology Glucocorticoid Hormone Action. SpringerVerlag, New York, pp 493-515

22. Greengard O 1970 The developmental formation of enzymes in rat liver. In: Litwack G (ed) Biochemical Actions of Hormones. Academic Press, New York, pp 53-87

23. Henning SJ 1981 Postnatal development: coordination of feeding, digestion, and metabolism. Am J Physiol 241:G199-G214 